\title{
Malária no Brasil: Panorama Epidemiológico na Última Década
}

\author{
Malaria in Brazil: Trends in the Last Ten Years
}

\author{
Rita de Cássia B. Barata ${ }^{1}$
}

\author{
BARATA, R. C. B. Malaria in Brazil: Trends in the Last Ten Years. Cad Saúde Públ., Rio de \\ Janeiro, 11 (1): 128-136, Jan/Mar, 1995.
}

There have been major changes in the epidemiological patterns for malaria in Brazil in the last ten years. One of the most important features is the tendency towards focal distribution in the Amazon region, instead of spreading transmission zones. This new epidemiological situation has led to changes in the control theory based on the occurrence of focal transmission of the infection. The importance of diversified control measures has thus increased, taking into account the characteristics of each transmission focus. The article comments on new proposals for control presented at the Ministerial Conference in Amsterdam.

Key words: Malaria; Epidemiology; Focal Transmission; Control Measures

\section{INTRODUÇÃO}

Os dados disponíveis sobre a ocorrência de malária, em todo o mundo, são bastante imprecisos em vista da baixa qualidade dos sistemas de registro de informações em saúde, principalmente na África, onde se concentram aproximadamente $80 \%$ dos casos clínicos da doença e $90 \%$ das infecções, atualmente. Estima-se que a cada ano cerca de 110 milhões de casos novos de malária e um a dois milhões de óbitos ocorram em todo o mundo. As maiores taxas de incidências são observadas na África, na Ásia e nas Américas. O número de indivíduos infectados é avaliado em 270 milhões de pessoas e a transmissão autóctone continua ocorrendo em 100 países (Najera et al., 1991; WHO, 1991).

Apesar desses números impressionantes, a malária é hoje uma doença focal, na maior parte do mundo. Apenas algumas regiões, em cada país, continuam apresentando transmissão natural da infecção. A situação atual difere marcadamente daquela que existia antes da Campanha de Erradicação, patrocinada

\footnotetext{
${ }^{1}$ Centro de Estudo Augusto Leopoldo, Departamento de Medicina Social. Faculdade de Ciências Médicas da Santa Casa de São Paulo. Rua Dr. Cesário Motta Júnior, 61, 6o andar, São Paulo SP, 01221-020, Brasil.
}

pela Organização Mundial da Saúde (OMS) com o apoio da Organización Panamericana de la Salud (OPS) e do governo norte-americano (através do Ponto IV e da Aliança para o Progresso), a partir de 1960, em todos os continentes. Naquele momento, a malária se apresentava como "doença de massa", atingindo vastas áreas e grandes contingentes populacionais em cada um dos países, onde a transmissão era endêmica.

\section{SITUAÇÃO EPIDEMIOLÓGICA}

No Brasil, após a realização da campanha de erradicação, durante a década de 60 , o número de casos de malária atingiu o seu valor mais baixo: 52.469 casos, confinando-se a transmissão, praticamente, à região amazônica. A manutenção da transmissão, após a campanha, foi atribuída à baixa densidade e à dispersão populacional na Amazônia, que dificultam a execução das ações de controle; ao tipo de habitação predominante nessa área que facilita os contatos homem-mosquito e atrapalha a aplicação de DDT; e ao aumento progressivo das cepas de Plasmodium falciparum resistentes à cloroquina, impedindo o esgotamento das fontes de infecção, com os recursos habitualmente utilizados (OPS, 1975). 
Na Amazônia, a ocorrência da doença também não é homogênea, havendo diferentes situações epidemiológicas, em função das diferentes formas de ocupação do solo e das diversas modalidades de exploração econômica dos recursos naturais. Os seringais, por exemplo, são áreas de baixa densidade populacional, com ocupação estável e pequena mobilidade, favorecendo o rociamento domiciliar com DDT e o uso de mosquiteiros, tornando a transmissão estável com baixa incidência. As áreas de pastagem, também apresentam incidências muito baixas, dado que a mão-de-obra é utilizada em pequeno número e a ausência da mata diminui a densidade dos vetores. Os acampamentos de construtoras são geralmente livres da malária em função do controle do ingresso dos trabalhadores, evitando a presença de portadores, controle de vetores e diagnóstico e tratamento precoces das fontes de infecção. Os garimpos fechados são praticamente livres de malária, dadas as condições ambientais desfavoráveis à transmissão. Os garimpos abertos, ao contrário, são áreas de alta incidência, dadas as facilidades de exposição, uma vez que os garimpeiros trabalham com pouca roupa e nos horários de maior atividade vetorial; há multiplicidade de criadouros decorrente da forma como o espaço é alterado pela presença do garimpo; e existência. de muitos portadores assintomáticos. As zonas novas de colonização têm incidências altíssimas, tendo em vista as condições favoráveis de transmissão, principalmente nas etapas iniciais em que a derrubada da mata é feita para o plantio, e a presença de inúmeros suscetíveis provenientes de áreas onde não há transmissão natural da doença. Já nas zonas de colonização antiga, a incidência tende a estabilizar-se, em níveis baixos, em decorrência da melhoria nas habitações, da presença de animais domésticos, diversificando as fontes alimentares para os anofelinos e das ações de controle praticadas. Os assentamentos espontâneos têm alta incidência, face ao rápido crescimento populacional dos núcleos urbanos nas áreas de garimpo ou nos projetos agrícolas. As áreas periurbanas de Manaus e Porto Velho apresentam incidências epidêmicas em função do constante afluxo de população. Face às dificuldades enfrentadas nas áreas de assentamento agrícola, essa população aban- dona suas terras e vem procurar emprego nas áreas urbanas. Acrescente-se a isto a multiplicação dos criadouros, provocada pela instalação das favelas. Finalmente, as aldeias indígenas que apresentam situação variável na dependência do maior ou menor contato com o "homem branco" infectado (WHO, 1988).

No início da década de $80,97,5 \%$ dos casos de malária registrados no Brasil eram produzidos na região amazônica. A ocupação desordenada da região, incentivada por diversos órgãos governamentais, a construção de estradas, de usinas hidroelétricas, o desenvolvimento de projetos agropecuários e a instalação de inúmeros garimpos provocaram o incremento considerável da transmissão (Marques et al., 1977; Marques, 1979; Rey, 1991; Sawyer \& Sawyer, 1987; Tauil, 1981, 1982). O número de casos novos de malária triplicou, passando de 52.469, em 1970, para 169.871, em 1980. Destes, 34,8\% ocorreram em Rondônia, 22,4\% no Pará, 11,3\% no Maranhão, 9\% no Mato Grosso e 8\% em Roraima.

Fora da Amazônia, o maior número de casos foi observado em Goiás, seguindo-se Paraná, São Paulo e Mato Grosso do Sul, estados que receberam a maior proporção de casos "exportados" pela região norte do país (Marques \& Pinheiro, 1982). Para as regiões indenes, a presença constante de indivíduos infectados, somado à persistência dos vetores, representa um risco contínuo de reintrodução da transmissão natural. A importância desses deslocamentos entre as áreas livres de transmissão e a área endêmica pode ser aquilatada pelos seguintes exemplos: apenas a zona de garimpo do projeto Cumaru "exportou" 8.683 casos para 381 municípios diferentes, em 11 estados. Os assentamentos agrícolas de Ariquemes produziram 1.134 casos, que foram diagnosticados em 204 municípios de dez estados (Botelho et al., 1988; Marques, 1986a, 1986b).

Em 1980 foram investigados cerca de 70 fluxos de casos entre os diferentes estados e os doze com maior intensidade (20 ou mais casos por mês) eram constituídos por correntes migratórias do sul do Pará e do norte do Mato Grosso para o Maranhão; do norte do Mato Grosso para São Paulo, Paraná, Goiás e Mato Grosso do Sul; e de Rondônia para São Paulo, Pará, Acre e Amazonas. 
Entre 1980 e 1985 houve um aumento de 2,4 vezes nos casos de malária com $63 \%$ deles, sendo produzidos em apenas 20 municípios da região amazônica, relacionados em sua maioria a projetos de assentamento agrícola e mineração. Nos projetos de colonização do Instituto Nacional de Colonização e Reforma Agrária (Incra) os colonos, no primeiro ano, derrubam três a quatro hectares de floresta e após a "queimada" plantam arroz, milho e feijão. O acampamento temporário, enquanto se procede à derrubada, é feito em ranchos de palmeiras ou barracas de plástico, armados nas proximidades do curso d'água, favorecendo a transmissão da malária, visto que com o desmatamento os vetores passam a se alimentar preferencialmente nos homens e as barracas não representam barreiras reais entre o "domicílio" e o ambiente externo. Após a "queimada" o sitiante constrói uma moradia provisória que dará lugar à casa definitiva geralmente feita de madeira (Sawyer \& Sawyer, 1987; Sawyer, 1989). Dos colonos que, em 1986, permaneciam no projeto Machadinho, $90 \%$ referiam ter tido malária, pelo menos uma vez, desde a sua chegada. Cerca de $56 \%$ referiam pelo menos cinco episódios e $71 \%$ apresentavam sorologia positiva para Plasmodium falciparum. A média horária de picadas de Anopheles darlingi era de 45,9, no peridomicílio, e o índice de infecção dos anofelinos capturados era de 5,2\%\% (Sawyer \& Sawyer, 1987; Sawyer, 1989).

Em 1990, todos os estados da Amazônia legal apresentaram índice Parasitário Anual (IPA) acima de sete lâminas positivas por 1.000 hab.: Maranhão (7,2 por 1.000), Amazonas (16,9 por 1.000), Pará (22,6 por 1.000), Mato Grosso (28,8 por 1.000), Acre (38,5 por 1.000), Amapá (43,2 por 1.000), Rondônia (128,3 por 1.000) e Roraima (146,5 por 1.000). A enorme disparidade de riscos entre os estados fica ainda mais acentuada quando se analisa a situação dos municípios. Cerca de 30 municípios foram responsáveis pela produção de $67 \%$ dos casos de malária, apresentando IPA de até 3.924 lâminas positivas por 1.000 habitantes (OPS, 1991).

No Acre, quatro dos doze municípios existentes respondem por $95 \%$ dos casos diagnosticados, cada um deles apresentando IPA acima de 100 lâminas positivas por $1.000 \mathrm{hab}$. Taxas altas de inci- dência são observadas nos assentamentos agrícolas e nos seringais do rio Abunã. No Amapá, apenas três municípios produzem $81 \%$ dos casos, todos eles com áreas importantes de garimpo. No estado do Amazonas somente três municípios apresentam IPA acima de 100 lâminas positivas por 1.000 hab., sendo que um desses municípios é o de Manaus onde a incidência de malária é epidêmica em decorrência da presença de grandes levas de migrantes, que abandonam suas roças e deslocam-se para a periferia da cidade, encontrando condições de saneamento básico propícias à multiplicação dos criadouros de anofelinos, instalando assim o ciclo de transmissão. No Mato Grosso, 16 municípios, todos eles com garimpo, apresentam altas taxas de incidência. No Pará, há 22 municípios, incluindo áreas de garimpo e colonização, em que o IPA é maior do que 100/1.000. Em Rondônia, 16 dos 23 municípios apresentam altas taxas de infecção, em áreas de colonização e garimpo. Finalmente, em Roraima, seis dos oito municípios apresentam IPA altos, todos eles ligados à presença de garimpos (Marques, 1992).

No início da década de 90, a malária no Brasil encontra-se confinada a 79 municípios da região amazônica, ligada, fundamentalmente, às atividades de mineração, nem sempre legais e passíveis de controle e, às atividades de expansão da fronteira agrícola, que parece estar atingindo as próprias fronteiras do país. Entretanto, tal "confinamento" não é completo, visto que, a partir dessas áreas relativamente restritas, os indivíduos infectados movimentam-se por todo o território, podendo reintroduzir a transmissão, em regiões onde ela já havia sido controlada.

$\mathrm{Na}$ última década foram registrados inúmeros focos, fora da região amazônica, provocados pela presença de indivíduos infectados, em localidades nas quais a densidade dos vetores é suficientemente alta para permitir o restabelecimento do ciclo. Normalmente a proporção desses surtos, secundários à introdução desses casos "importados", varia inversamente com o estágio do desenvolvimento socioeconômico e, diretamente, com o sistema de vigilância epidemiológica existente. Assim, nas áreas mais desenvolvidas e que também possuem os melhores sistemas de vigilância, a introdução de casos importados costuma ser prontamente diagnosticada, evitando-se a produção de um foco. Quando tal diagnóstico não 
é feito, surgem alguns casos "introduzidos" que, uma vez identificados, determinam a adoção de medidas de contenção de foco, limitando o número de casos secundários. Nas áreas com menor desenvolvimento e com piores condições para a vigilância epidemiológica, a demora na detecção de casos importados pode propiciar a instalação de focos com maiores dimensões, isto é, com número relativamente grande de casos introduzidos.

Verifica-se, assim, que a malária se constitui hoje em um problema localizado e relacionado com a exploração das riquezas minerais em garimpos abertos e com a ocupação agrícola das terras da região norte, originalmente cobertas pela floresta amazônica. Relativamente poucas localidades respondem pela produção da maioria dos casos. São de grande importância para o controle da doença, os deslocamentos freqüentes de indivíduos que, por razões ocupacionais principalmente, se movimentam entre a área endêmica e as diversas áreas indenes do país.

\section{CONHECIMENTO SOBRE MALÁRIA}

A reversão das expectativas com relação à possibilidade de erradicação da malária, no prazo de poucos anos, bem como o agravamento de algumas situações quanto à resistência dos parasitas aos antimaláricos e dos vetores aos inseticidas, determinaram, no campo do conhecimento cientifico, dois tipos de movimento, a partir da década de 70. Por um lado, intensificaram-se as pesquisas clínicas e biológicas, buscando aprofundar e detalhar o conhecimento relativo ao processo infeccioso, visando melhor caracterizar as diferentes etapas do processo de transmissão; e, por outro lado, promoveu-se a revisão dos conceitos e estratégias na luta contra a doença, reconhecendo-se seu caráter predominantemente focal.

Os estudos entomológicos mostraram, por exemplo, que a infectividade dos esporozoítas não é constante durante o ciclo de vida do vetor, caindo significantemente a partir da terceira semana, assinalando assim a importância dos portadores nas fases iniciais da infecção. As diversas espécies de plasmódios também têm infectividade variável, decrescendo do P. falciparum para o P. ovale, conhecimen- to importante para a compreensão da fórmula parasitária. Os estudos parasitológicos mostraram que parte das formas exoeritrocitárias permanecem quiescentes no organismo, originando as recaídas. Esses estudos permitiram, ainda, a verificação de que o $P$. malarie infecta tanto homens quanto macacos, podendo manter a transmissão, em situações particulares (Rey, 1991). A resistência dos plasmódios às drogas também passou a ser bastante investigada a partir da padronização e divulgação dos testes in vitro e dos critérios estabelecidos pela OMS para a classificação da resistência in vivo, elementos importantes na condução dos tratamentos (OMS, 1986). As investigações imunológicas, além de buscarem novas técnicas diagnósticas mais sensíveis e específicas, permitiram avançar no conhecimento dos mecanismos de defesa do hospedeiro e estabelecer a importância dos portadores. A imunidade ativa natural parece estabelecer-se lentamente, após quatro ou cinco infecções para $P$. vivax, e dez ou mais para $P$. falciparum, havendo a possibilidade da imunidade ser suficiente para suprimir os sintomas e sinais clínicos, sem, contudo, interferir na formação dos gametas, mantendo, assim, as fontes de infecção na comunidade (Clyde, 1989).

O recrudescimento da malária em várias partes do mundo, inicialmente foi visto como decorrência das falhas técnicas e operacionais na condução das campanhas, afiadas à redução dos recursos financeiros destinados à erradicação e a um certo arrefecimento na decisão política (Bruce-Chwatt, 1986; Lepes, 1981). Diante da rápida deterioração da situação epidemiológica, porém, essa tese foi perdendo força e progressivamente foi sendo substituída pela teoria focal da doença.

Frente à variedade, desconcertante, de formas assumidas pela malária, nos diversos contextos situacionais, alguns autores elaboram "paradigmas" que buscam reunir os determinantes ecológicos e sociais em certos "tipos ideais". Najera, distribui seus "paradigmas" em dois conjuntos: condições ecológicas específicas e condições ocupacionais e/ou sociais específicas.

Os primeiros compreendem: malária da savana africana, malária de vales e planícies fora da África, malária de florestas, malária de desertos 
ou áreas altas, malária dos pântanos e áreas costeiras. Entre os segundos temos: malária dos projetos de colonização agrícola em áreas de floresta, malária de mineração, malária de acampamentos de trabalhadores migrantes ou campos de refugiados (Najera et al., 1991).

Os paradigmas tentam retratar, com algum nível de generalidade, as situações concretas de persistência da transmissão ou de reintrodução da malária que só se tornaram evidentes após a execução da campanha de erradicação, seja porque se apresentam como "situações residuais" resistentes às estratégias de controle adotadas, seja porque não existiam anteriormente, vindo a se constituir no processo de desenvolvimento histórico e econômico de alguns países. Abandona-se a concepção que atribuía ao "fracasso" das campanhas o ressurgimento da malária e seu crescimento na década de 80 , substituindo-a pela idéia de "foco". Passa-se, assim, de uma perspectiva negativa de fracasso, para outra, plenamente afirmativa, de situações peculiares (os focos) que necessitam de abordagens específicas.

\section{PROPOSTAS PARA O CONTROLE}

O reconhecimento da impossibilidade de erradicação, em várias partes do mundo, levou a Organização Mundial da Saúde a propor a transformação dos programas de erradicação em programas de controle, já no início da década de 70 (OMS, 1971). O 15 Informe do Comite de Expertos em Malária sugeriu aos diferentes países que, em vista de suas situações epidemiológicas, adotassem um entre três tipos de programas: programas nacionais de erradicação com duração limitada; programas de erradicação, desenvolvidos em etapas sucessivas, cobrindo progressivamente o território; ou programas de controle a longo prazo.

Além da modificação na estratégia os informes do Comitê propõem a adoção de medidas diversificadas de controle, abrangendo: a luta antivetorial, incluindo a prevenção da formação dos criadouros, uso de larvicidas, combate biológico, uso de inseticidas residuais; a prevenção dos contatos homem-vetor, através de medidas de proteção individual, tais como o uso de mosquiteiros, telagem, repelentes, roupas proteto- ras etc.; a quimioprotilaxia em massa para grupos de alto risco (gestantes e crianças em áreas hiperendêmicas). Desta forma a conduta padronizada da campanha, baseada exclusivamente na dedetização dos domicílios e na busca ativa e tratamento presuntivo dos casos febris, dá lugar a uma diversidade de abordagens que visa adequar a proposta de intervenção às características epidemiológicas de cada região (Ayalde, 1976; Schliessman, 1974). A maioria dos países, entretanto, teve dificuldade em modificar, efetivamente, seus programas de erradicação e em 1977 nova mudança é proposta pela OMS, no sentido de que o controle da malária passe a fazer parte das atividades de assistência primária, sem, entretanto, obter grande sucesso (OPS/OMS, 1977).

Nova tentativa de redirecionar os programas é feita por ocasião da publicação do $17^{\circ}$ Informe. A técnica de estratificação epidemiológica é proposta como instrumento de caracterização do comportamento focal da endemia e para orientar a escolha de práticas de controle específicas para cada localidade (OMS, 1979, 1986; Yang, 1986). São propostas as quatro variantes táticas elaboradas para dar conta de diferentes situações. A variante um tem por objetivos a redução e a prevenção da mortalidade, baseando-se na distribuição de medicamentos antimaláricos pelos serviços básicos de saúde. A variante dois visa à prevenção da mortalidade e à redução da morbidade em grupos de alto risco, através do diagnóstico e tratamento precoce e de programas de quimioprofilaxia associados ou não à adoção de medidas de proteção individual. A variante três busca a redução da prevalência da infecção, combinando medidas de combate aos vetores e controle das fontes de transmissão. Finalmente, a variante quatro corresponde aos programas nacionais de erradicação (OMS, 1979).

No Brasil, as medidas adotadas no sentido de adequar o programa às dificuldades encontradas, passaram, inicialmente, pela divisão do país em duas áreas: a de erradicação a curto prazo e a de erradicação a longo prazo (Amazônia), sem que houvesse mudanças substanciais na forma de aplicação das medidas de controle.

Durante a década de 80 , toda a atenção da Superintendência de Campanhas de Saúde Pública (Sucam) será concentrada na Amazônia, principalmente naquelas localidades com si- 
tuações especiais de risco: construção de hidroelétricas, garimpos, projetos de assentamento agrícola e outras (Deane, 1988; Sucam/MS, 1981a, 1981b). Em muitos garimpos a situação mostra-se refratária às medidas de controle, tendo em vista as habitações precárias, o movimento constante de entradas e saídas de garimpeiros e o altíssimo custo de vida, inviabilizando a permanência, nessas áreas, dos funcionários da Sucam. Nas áreas de projetos agropecuários e de grandes obras de engenharia, a Sucam assume a responsabilidade pela aplicação semestral de DDT, orientação para eliminação de criadouros, treinamento de microscopistas, fornecimento de medicamentos antimaláricos e supervisão técnica, ficando o trabalho de controle por conta das próprias empresas (Sucam/MS, 1981b). Há vários exemplos de sucesso como a construção da hidroelétrica de Balbina, ferrovia Carajás-Itaiqui, rodovia Cuiabá-Porto Velho, e outros exemplos negativos, como a construção de Tucuruí, os projetos de colonização do Incra, as minas de ouro de Itaituba e do rio Madeira (Chagas et al., 1982; Tauil, 1986).

Apesar das modificações introduzidas na condução do programa a incidência continuou aumentando durante a década de 80 , levando à admissão da necessidade de abandono da estratégia de erradicação e da elaboração de projetos locais (Tauil et al., 1985). A Sucam adota, então, a estratificação epidemiológica e a microzonagem, incorporando técnicas mais diversificadas de controle que incluem as nebulizações a ultrabaixo volume, tratamento em massa, cortinas impregnadas e novos inseticidas. Entretanto, em muitas áreas como nas linhas de colonização, em Rondônia, as ações de controle permanecem inalteradas apesar da evidente ineficiência. Os colonos não acreditam na eficácia do DDT, pois as paredes de suas casas são incompletas e a maior densidade de mosquitos é observada no peridomicílio. O tratamento supressivo também é desacreditado e o lapso de tempo, geralmente grande, entre o diagnóstico (coleta do sangue) e o início do tratamento radical leva ao abandono da medicação antes que o tratamento se complete (Dias et al., 1991). Mesclam-se atividades novas e a manutenção dos velhos hábitos, fortemente arraigados, nos funcionários, que viveram a época da campanha de erradicação.
Desde 1989 os casos de malária vêm diminuindo, em parte, graças à estabilização da incidência nas áreas de colonização, passada a etapa inicial de ocupação e, em parte, graças às mudanças adotadas na estratégia de controle, que conferiram maior autonomia às direções locais, buscando adaptar os instrumentos disponíveis às diversas situações epidemiológicas. Esta nova atitude, entretanto, encontra seu limite na escassez de profissionais devidamente capacitados para a avaliação da estrutura epidemiológica e a organização de uma proposta coerente de intervenção. A padronização extrema das condutas, vigente durante a fase campanhista, dificulta, agora, uma atitude mais flexível por parte dos profissionais. Agrega-se a esta dificuldade a desorganização do aparelho do Estado no nível federal e, em grande medida, também no nível estadual, e a indefinição dos papéis desses dois níveis face às propostas de municipalização.

O controle das endemias, e, dentre elas, da malária, encontra-se em um período de transição, no qual os diferentes papéis institucionais não se encontram claramente explicitados. Diversas experiências têm sido feitas no sentido de concretizar a transferência das atribuições para as instâncias regionais, discutindo-se ainda a possibilidade dos governos estaduais assumirem as estruturas de controle existentes em seus territórios. Tais transferências ainda não foram concretizadas impossibilitando uma avaliação de seu impacto no programa de controle. Entretanto, tem crescido, nas áreas mais afetadas pela endemia, a participação das prefeituras municipais, nas ações de controle, visando suplementar as atividades desenvolvidas pela Fundação Nacional de Saúde.

\section{MOMENTO ATUAL}

A tendência apresentada pela última conferência ministerial promovida pela OMS em Amsterdan, 1992, aponta para o abandono das estratégias coletivas no combate à malária e para o privilegiamento do enfoque de risco, valorizando mais as intervenções sobre os indivíduos e menos as intervenções sobre o meio ambiente. 
Segundo as diretrizes aprovadas pelos ministros da saúde, o controle da malária, nos próximos anos, deve estar baseado em três conjuntos de atividades. O primeiro conjunto é designado de gestão da doença e inclui as atividades relativas ao diagnóstico e ao tratamento dos casos. Os governos devem garantir os meios para que os indivíduos doentes obtenham o diagnóstico correto e o tratamento adequado e oportuno de sua doença. Não se trata mais de eliminar fontes de infecção, mas de reduzir a morbidade e a mortalidade por malária. As atividades de busca ativa devem ser abandonadas e a "gestão da doença" deve ser atribuída aos serviços de assistência primária. O segundo conjunto de atividades é o da prevenção da doença, que inclui medidas de proteção individual, quimioprofilaxia, imunização e controle domiciliar dos vetores. $\mathrm{O}$ foco das ações de controle desloca-se do ambiente e das fontes de infecção para a proteção dos possíveis hospedeiros, dirigindo-se para os indivíduos e seus "comportamentos de risco". O terceiro conjunto de atividades destina-se à prevenção e controle de epidemias, incluindo as ações tradicionais de vigilância epidemiológica, retendo o caráter mais coletivo da intervenção e maior responsabilidade do Estado em sua execução (OMS, 1992).

Transferir para os indivíduos a responsabilidade por sua própria proteção, restando aos programas a tarefa de garantir o fornecimento dos recursos necessários para o diagnóstico e o tratamento, pode determinar o agravamento das inequiidades. As ações coletivas atingem todos os indivíduos, estejam ou não realmente sujeitos a desenvolver a doença, porém, em sua ação indiscriminada, alteram as condições de produção da doença, independentemente de comportamentos individuais, agindo sobre a sociedade mais do que sobre cada sujeito. As ações individuais, por seu turno, atuam sobre cada um dos sujeitos que as adotam, sem alterar a situação geral. Assim, as ineqüidades, presentes na organização social, tendem a se reproduzir nas práticas de controle, uma vez que, os sujeitos mantidos à margem na organização social, e que geralmente são os que apresentam maior risco de adoecer, também terão maiores dificulda- des para utilizar as medidas de proteção preconizadas (Bradley, 1991).

Desta forma o problema do controle é transferido da esfera da produção, onde os determinantes estruturais do processo saúde-doença atuam, para a esfera do consumo de medidas e práticas preventivas, com todas as características de ineqüidade aí existentes, nas formações sociais capitalistas. A nova estratégia de controle, praticamente, desiste da pretensão de tornar acessível a todos, os avanços da ciência e da técnica, assumindo a realidade das desigualdades socioeconômicas e a impossibilidade de minorá-las, via intervenções técnicas. O pragmatismo e a capitulação diante das dificuldades em estender o controle para aquelas regiões onde es condições de desenvolvimento socioeconômico são muito desfavoráveis, presentes na atual estratégia, mostram-se perfeitamente coerentes com a "nova ordem mundial" onde os "ineptos", para acumular capital em seu próprio benefício, são deixados à margem do sistema, acumulando desigualdades sociais profundas e problemas insolúveis.

\section{RESUMO}

\section{BARATA, R. B. Malária no Brasil: Panorama Epidemiológico na última Década.} Cad. Saúde Públ., Rio de Janeiro, 11 (1): 128-136, jan/mar, 1995.

Este artigo apresenta, de maneira resumida, as principais alterações que marcaram a ocorrência de malária, no Brasil, nesta última década. Chama-se a atenção para o processo de focalização da doença, com a concentração da transmissão em áreas, relativamente restritas, da região amazônica. A mudança na situação epidemiológica acarreta a revisãoconceitual, levando ao abandono da concepção de "doença de massa" e à elaboração da teoria focal. Do mesmo modo, as ações de controle sofrem os impactos das transformações ocorridas e as tentativas de erradicação são abandonadas e substituídas por medidas de controle diversificadas e adaptadas a cada localidade. As novas 
propostas, oriundas da Conferência Ministerial de Amsterdam, são apresentadas e comentadas.

Palavras-Chave: Malária; Epidemiologia; Transmissão Focal; Medidas de Controle

\section{REFERÊNCIAS BIBLIOGRÁFICAS}

AYALDE, J., 1976. Analisis de Ia estrategia en uso de tos programas de erradicación de la mataria en las Americas. Boletin de la Oficina Sanitaria Panamericana, 80: 45-51.

BOTELHO, C.; BARBOSA, L. G. S.; SILVA, M. D. \& MEIRELLES, S. M. P., 1988. Fluxo migratório de casos de malária em Cuiabá, MT, 1986. Revista do Instituto de Medicina Tropical, 30: 212-220.

BRADLEY, D. J., 1991. Malaria - whence and whiter? In: Malaria: Waiting for the Vaccine (G. A. T. Targett, ed.), pp. 11-29, London: London School of Hygiene and Tropical Medicine.

BRUCE-CHWATT, L., 1986. Malaria Control at the Cross-roads! Where do we go from here? Memórias do Instituto Oswaldo Cruz, 81 (supl. II): 0104.

CHAGAS, J. A. C.; BARRoSO, M. A. B.; AMORIN, R. D. S. \& ROBLES, C. R. Q., 1982. Controle da malária em projeto hidroelétrico no Estado do Amazonas. Revista Brasileira de Malariologia e Doenças Tropicais, 34: 58-81.

CLYDE, D. F., 1989. Epidemiologic significance of immunity in vivax malaria. Epidemiologic Reviews, 11: 109-125.

DEANE; L., 1988. Mataria studies and control in Brazil. American Journal Tropical Medicine and Hygiene, 38: 223-230.

DIAS, R. P.; MACHADO, C. F. S. \& PEIXOTO, M. C. D., 1991. Cotidiano de Vida em Áreas de Colonização da Amazônia e Estratégia de Controle da Malária. Belo Horizonte, Pontifícia Universidade Católica. (Mimeo.)

LEPES, T., 1981. Malaria. A Global Health Problem. WHO/MAL/81.949. Genebra: WHO. (Mimeo.)

MARQUES, A. C., 1979. Migrações internas e grandes endemias. Revista Brasileira de Malariologia e Doenças Tropicais, 31: 137-158.

1986a. Migrations and dissemination of mataria in Brazil. Memórias do Instituto Oswaldo Cruz, 81 (supl. II): 39-41.

1986b. Um estudo sobre a dispersão de casos de malária no Brasil. Revista Brasileira de Malariologia e Doenças Tropicais, 38: 51-75.
1992. Situação Epidemiológica da Malária no Brasil. 2o Congresso Brasileiro de Epidemiologia. Belo Horizonte: Abrasco.

MARQUES, A. C. \& PINHEIRO, E. A., 1982. Fluxos de casos de malária no Brasil em 1980. Revista Brasileira de Malariologia e Doenças Tropicais, 34: 01-31.

MARQUES, A. C.; PINHEIRO, E. A. \& MOTTA, E. G. F., 1977. Fatores que Dificultam o Controle da Malária na Região Amazônica. XIX Congresso Brasileiro de Higiene, Rio de Janeiro. (Mimeo.)

NAJERA, J.; LIESE, B. \& HAMMER, J. S., 1991. Health Sector Priorities Review: Malaria. Washington, DC: The World Bank.

OMS (Organización Mundial de la Salud), 1971. Comité de Expertos en Malaria. 15음 Informe. Genebra: OMS. (Serie Informes Técnicos, 467)

1979. Comite de Expertos en Malaria. $17^{\circ}$ Informe. Genebra: OMS. (Serie de Informes Tecnicos, 640)

1986. Comite de Expertos en Mataria. $18^{\circ}$ Informe. Genebra: OMS. (Serie de Informes Tecnicos, 735)

1992. Estrategia Mundial de la Lucha Contra el Paludismo. Conferencia Ministerial, Amsterdan: OMS. (Mimeo.)

OPS (Organización Panamericana de la Salud), 1975. Reunião de Diretores de Serviços de Erradicação da Malária das Américas. Washington, DC: OPS. (Mimeo.)

1991. Estratificação epidemiológica da malária na região das Américas. Boletim Epidemiológico, 12: 01-04.

OPS/OMS (Organización Panamericana de la Salud/ Organización Mundial de la Salud), 1977. El Control de la Mataria en las Americas. Relatório Técnico. Washington, DC: OPS.

REY, L., (Org.) 1991. Parasitologia. Rio de Janeiro: Guanabara-Koogan.

SAWYER, D., 1989. Malaria on the Amazon Frontier: Integrated Participation for Control of Malaria in a New Agricultural Settlements. Belo Horizonte: Cedeplar.

SAWYER, D. \& SAWYER, D. R. T. O., 1987. Malaria on the Amazon Frontier: Economic and Social Aspects of Transmision and Control. Belo Horizonte: Cedeplar.

SCHLIESSMAN, D. J., 1974. Technical and Economic Justification for Use of Comprhensive Antimosquito Measures in Mataria Control and Eradication Programmes. WHO/MAL 74.835. Genebra: WHO. (Mimeo.) 
SUCAM/MS (Superintendência de Campanhas de Saúde Pública/Ministério da Saúde), 1981a. Padronização de Ações de Combate em Áreas de Exploração de Garimpo. Brasília, DF: MS.

, 1981b. Requisitos e Responsabilidades da Sucam e de Empresas para a Proteção contra Malária, Febre Amarela e a Leishmaniose Tegumentar. Brasília, DF: MS. (Norma Técnica)

TAUIL, P. L., 1981. Estudo de Alguns Aspectos da Epidemia de Malária em Porto Nacional (GO) Brasil. Goiânia: UFGO.

1982. Malária: Situação Atual no Brasil: Problemas e Perspectivas do Programa de Erradicação. XVII Congresso da Sociedade Brasileira de Medicina Tropical, Ribeirão Preto. (Mimeo.)

1986. Comments on the epidemiology and control of malaria in Brazil. Memórias do Instituto Oswaldo Cruz, 81 (supl. II): 39-41.
TAUIL, P. L.; DEANE, L.; SABROZA, P. C. \& RIBEIRO, C.,1985. A malária no Brasil. Cadernos de Saúde Pública, 1: 71-111.

WHO (World Health Organization), 1988. Report on a Technical Consultation on Research in Support of Malaria Control in the Amazon Basin. TDR/ FIELD MAL/SC/AMAI 88.3. Genebra: WHO. (Mimeo.)

1991. World Health Statistics Annual. Genève: WHO.

YANG, C., 1986. Epidemiological Diversity and Selection of Measures for Malaria Control within the Strategy of Primary Health Care: Some Experiences in China. WHO/MAL 86.1030. Genebra: WHO. (Mimeo.) 